\title{
Face Detection using Principal Component Analysis (PCA)
}

\author{
Pushpak Dave \\ M.Tech, EC \\ TIT, Bhopal
}

\author{
Jatin Agarwal \\ M.Tech, EC \\ TIT, Bhopal
}

\author{
Tarun Metta \\ Research Scholar \\ E\&TC Department \\ Amity University
}

\begin{abstract}
Face Detection makes it possible to use the facial images of a person to authenticate him into secure system, for criminal identification, for passport verification etc. It is done by Principal Component Analysis (PCA).Face images are projected onto a face space that encodes best variation among known face images. The face space is collection of Eigen face. In the algorithm, initially video segmented using shot boundary detection techniques. Specifically, it can detect both the cut and gradual shot transitions in video. For detecting the shot boundary haar wavelet transform is used. In this method, each frame and its haar wavelet transform image is correlated for detection the shot. By setting the threshold of frame correlation shot boundaries can be detected. Video segmentation can be used in various application like video summarization, video search, and video annotation.
\end{abstract}

\section{General Terms}

For detecting face there are various algorithms including skin color based algorithms like Image acquisition, get video frame, run detector, bonding box around face etc.

\section{Keywords}

Face detection, PCA, Eigen Face, Shot boundary, Haar wavelet transform

\section{INTRODUCTION}

Face detection is used in many places now days especially the websites hosting images like Picasa, photo bucket and Facebook. The automatically tagging feature adds a new dimension to sharing pictures among the people who are in the picture and also gives the idea to other people about who the person is in the image.

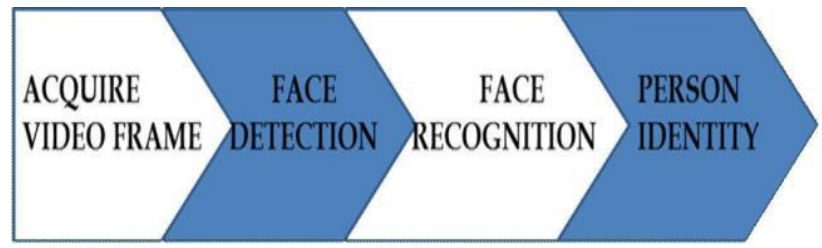

Fig 1: Face Recognition System

Face and non-face images are described in terms of wavelet feature in AdaBoost method. Principal Component Analysis (PCA) is used to generate the feature vector of face and nonface image in Eigen Face method. Also, PCA is used to compress the given information vector. Face detection algorithm gives location of face or face area with facial features (eye, nose, lips etc).The face is our primary focus of attention in social life playing an important role in conveying identity

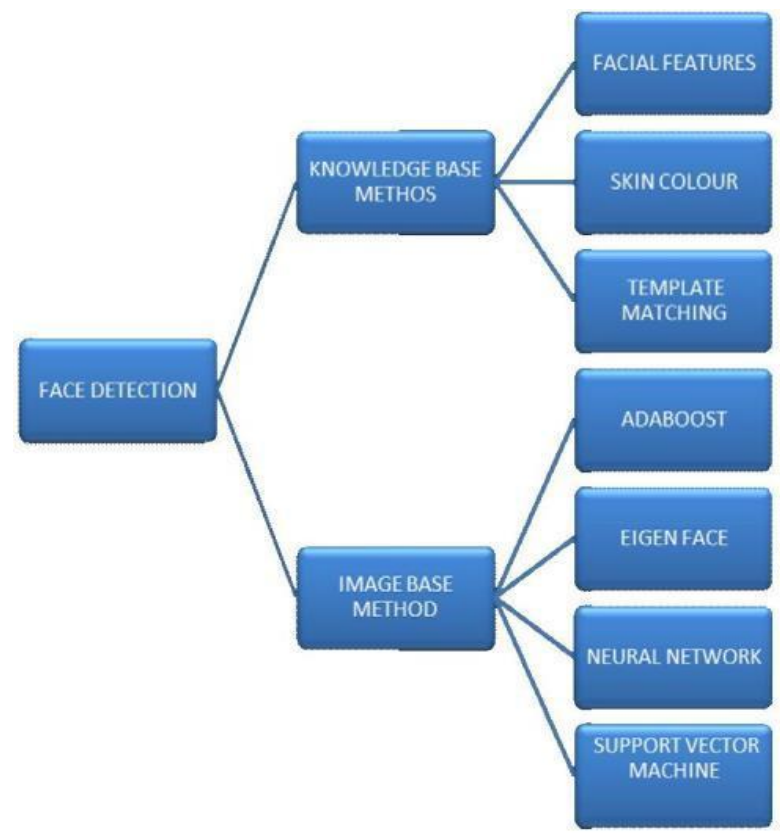

Fig 2: Face Detection System

PCA is used to compress the given information vector. Kernel function is created to describe face and non-face images in Support Vector Machines (SVM). Significant information about the human face detection is pattern of human face. Template matching can be applied over window scanning technique or segmented region.

\section{SHOT BOUNDARY DETECTION}

Text must be single-spaced using a Times New Roman font, Use a 24-point font for the Title, a 10-point font for Author Name(s), a 9-point font for Affiliation(s), a 10 point for all body text and all Section and Subsection Heads.

Generally a video can be structured in a hierarchical form as "videos $\rightarrow$ shots $\rightarrow$ Scene $\rightarrow$ key frames".

The amount of multimedia data has grown significantly in the past few years. This growth is primarily due to advances in data acquisition, storage, and communication technologies, aided by advances in processing of audio and video signals. Video has played an important role in this growth, more so in terms of its volume. The exponential growth of visual data in digital format has led to a corresponding search problem. 


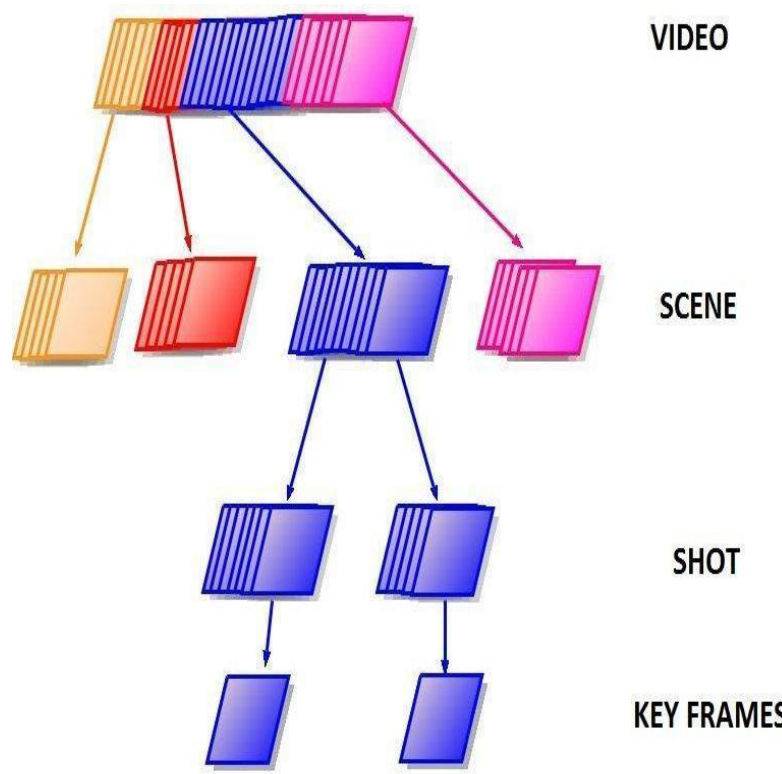

Fig 3: Structure of Video File

\subsection{Haar Wavelet Transform}

Wavelet theory is applicable to several subjects. All wavelet transforms may be considered forms of timefrequency representation for continuous-time (analog) signals and so are related to harmonic analysis. Almost all practically useful discrete wavelet transforms use discrete-time filter banks.

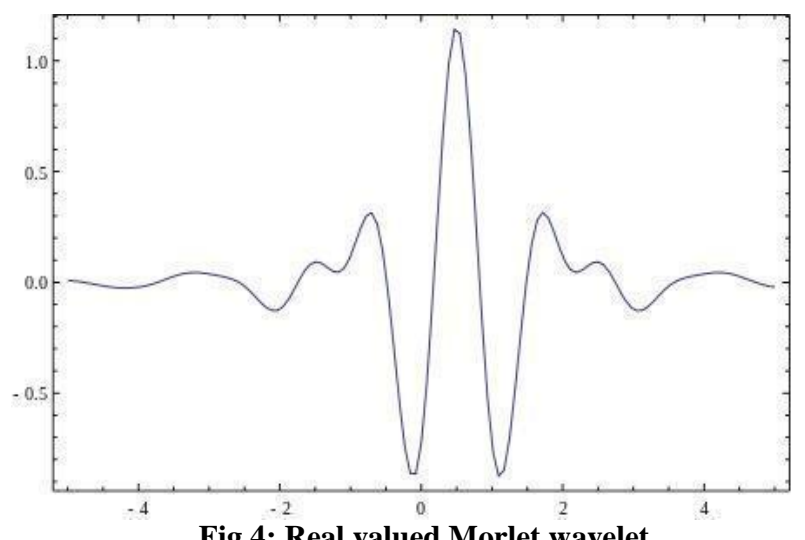

Fig 4: Real valued Morlet wavelet

A wavelet is a wave-like oscillation with an amplitude that starts out at zero (0), increases, and then decreases back to zero. It can typically be visualized as a "brief oscillation" like one might see recorded by a seismograph or heart monitor. The product of the uncertainties of time and frequency response scale has a lower bound. Wavelets can be combined, using a "reverse, shift, multiply and sum" technique called convolution, with portions of an unknown signal to extract information from the unknown signal. Wavelet theory is applicable to several subjects. All wavelet transforms may be considered forms of time-frequency representation for continuous-time (analog) signals and so are related to harmonic analysis. Almost all practically useful discrete wavelet transforms use time filter.

\section{ALGORITHM OF SHOT DETECTION AND KEY FRAME EXTRACTION}

A shot consists of a continuous sequence of video frames recorded by an uninterrupted camera operation and consistent background settings, and a scene can be considered as a group of semantically related shots which represents different views of the same theme and contains the same object of interest. each shot key frame is extracted [10].Ix=I1,I2...IN. denote the images of the video clip V. Position of the shot can be find from following step.

Step 1: Take each frame from video and divide in to8x8 sub image.

Step 2: For each sub image calculate variance ofintensityVli. And find haar wavelet transform of each sub image. Also calculate variance of intensity of that sub image denote as Vwi.

Step3: Calculate mean of intensity variance VLAN of overall nth image and also calculate mean of intensity variance VWAN of overall haar wavelet transform image.

Step 4: Calculate integrated coefficient IC of the nth image $I C^{i} \quad\left(\left|V L^{i} \quad V L A N\right|,\left|V W^{i} V W A N\right|\right)(1)$

Step 5: ${ }^{n}$ calculate ${ }^{n}$ Inter-image correlation between two consecutive images, $\mathrm{n}$ and $(\mathrm{n}+1)$ is defined

$\mathrm{FC}(\mathrm{n}, \mathrm{n}+1) \min =\quad\left(I C_{n}{ }^{i}, I C_{n}{ }^{i} \quad 1\right)$

Step 6: The threshold of the inter-image correlation isset at Tshot. Namely, image $\mathrm{n}$ can be considered as a shot transition point if following inequalities are simultaneously satisfied:

$$
\begin{aligned}
& F C(n)-F C(n-1)<-T \text { shot } \\
& F C(n+1)-F C(n)>T \text { shot }
\end{aligned}
$$

\subsection{Key Frame Extraction}

After detecting shot boundaries, each shot can now be represented using a characteristic set of either individual frame or short sequences instead of all the frames within a shot.

The key frames are obtained by the following steps:

Step 1: The inter-frame correlation within the sameshot is formulated as below:

$$
\operatorname{IFC}\left(\mathrm{I}_{j} \mathrm{I}_{1}\right)=\frac{\sum_{i}\left|I C_{j}^{i}-I C_{l}^{i}\right|}{\sum_{i} \min \left(\left|I C_{j}^{i}\right|_{\imath}\left|I C_{l}^{i}\right|\right)}
$$

Step 2: Let Ss = $(\mathrm{f} 1, \mathrm{f} 2 \ldots \mathrm{fL})$ denote the frames of video shot $\mathrm{S}$, and frame $\mathrm{f} 1$ is always chosen as the first key frame: $\mathrm{KS}=\left\{K_{1}^{s}\right\}=\{\mathrm{f} 1\}$

Step 3: For the current frame $\mathrm{Ij}$ and the set of keyframes $\mathrm{Ks}=$ $\{\mathrm{Ks} 1, \mathrm{Ks} 2 \ldots \mathrm{Ksk}\}$, if Min (IFC (Ij, Ii)) > Tkeyframe, then Ij can be absorbed into the key frame set.

\section{FACE DETECTION AND RETRIEVAL}

The goal is to take a Video and indicate the location of faces 
as in that video. Detection of faces in digital image has gained much importance in the last decade, with applications in fields such as law enforcement and security.

\subsection{Why Face Detection is Important}

Face Detection is a computer based technology to determine the location of a face( or faces) in an image regardless of its size, color, illumination and ignoring all other constituents of the image.

\subsection{Different Color Tones}

The study on skin color classification has gained increasing attention in recent years due to the active research in contentbased image representation.

\subsubsection{RGB Mode (Red Green Blue)}

The RGB color model is an additive color model in which red, green, and blue light are added together in various ways to reproduce a broad array of colors.

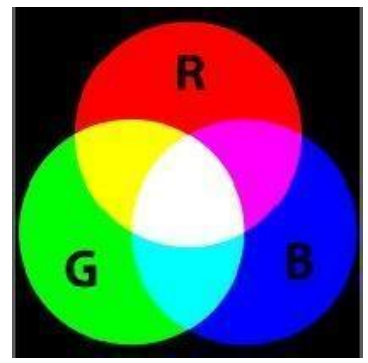

\section{Fig 5: Real valued Morlet wavelet}

The RGB model can be represented by a 3-dimensional cube in 3-D space with its center at the origin and $R, G, B$ components along the three axes.

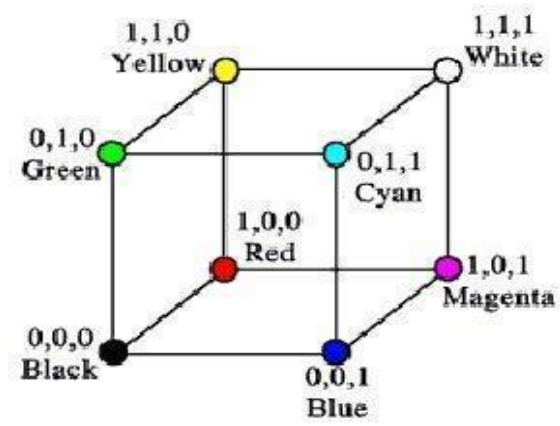

Fig 6: Real valued Morlet wavelet

\subsubsection{YCbCr Mode (Yellow Blue Difference Red Difference)}

$\mathrm{YCbCr}$ color space has been defined in response to increasing demands for digital algorithms in handling video information, and has since become a widely used model in a digital video. These color spaces separate RGB (Red-Green-Blue) into luminance and chrominance components. $\mathrm{Y}$ is the luminance and $\mathrm{Cb}$ and $\mathrm{Cr}$ are the blue-difference (Blue Luminance) and red-difference (Red- luminance) chroma components. $\mathrm{YCbCr}$ is not an absolute color space. The actual color displayed depends on the actual RGB colorants used to display the original image. $\mathrm{YCbCr}$ mode is used for image compression work

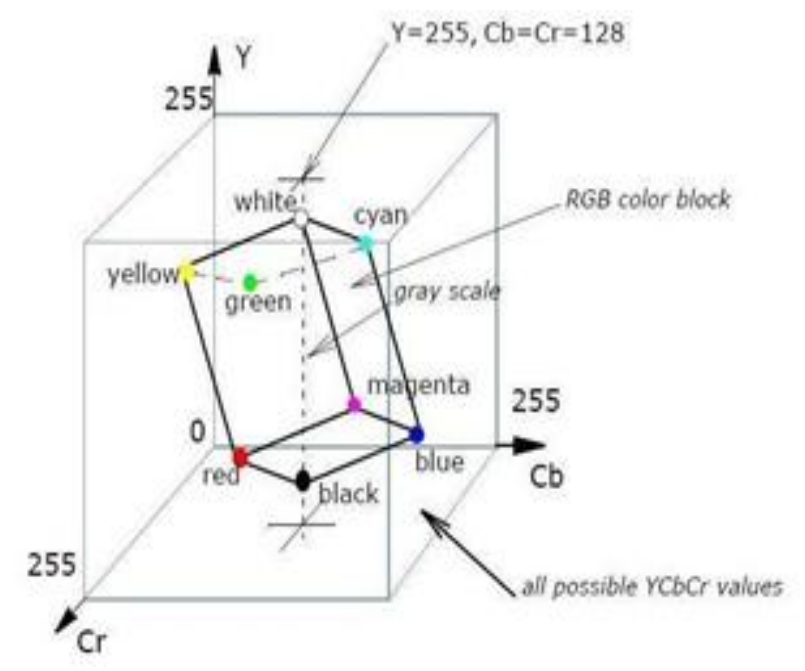

Fig 7: Real valued Morlet wavelet

\subsubsection{HSV/HSI Mode}

HSV stands for hue, saturation and value. Hue is one of the main properties of a color. It represents a pure color (without any tint or shade). A hue is an element of the color wheel. Saturation is the difference of a color against its own brightness.The saturation of a color is determined by a combination of light intensity and how much it is distributed across the spectrum of different wavelengths.

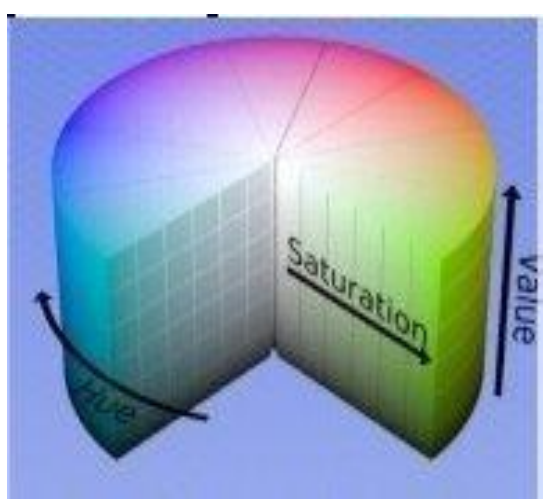

Fig 8: Real valued Morlet wavelet

\subsubsection{Algorithm For Face Detection}

For detecting face there are various algorithms including skin color based algorithms. Color is an important feature of human faces. Using skin-color as a feature for tracking a face has several advantages. 


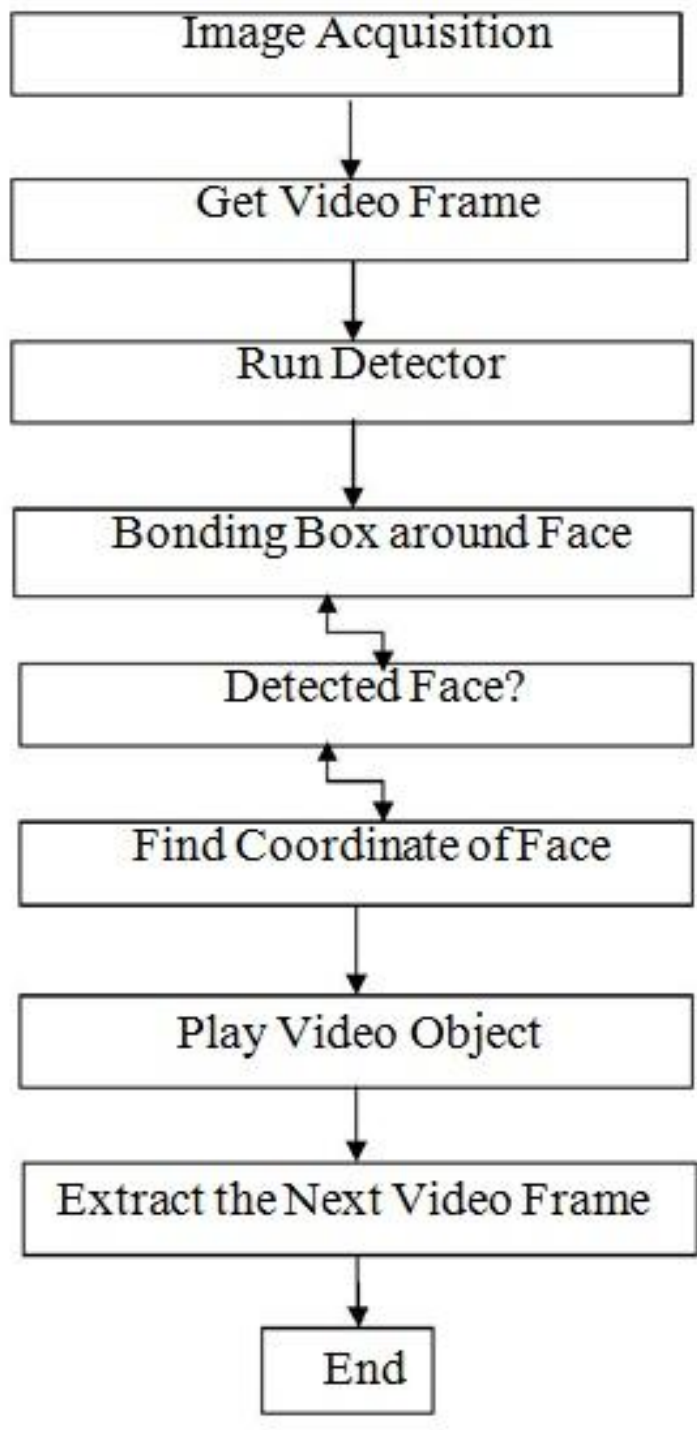

Fig 9: Real valued Morlet wavelet

\section{CONCLUSION}

In this review paper, algorithm that is used for shot detection that detects cut and gradual shot successfully. This algorithm used haar wavelet transform that is most effective for deciding the boundary of shot.

Presented a face detection algorithm can successfully detected a face in video. That combines pixel from different color tones. According to threshold value of each color model, skin area is detected. Detected skin area is decided whether it is face or not. For finding the face in skin area length to width ratio of each connected skin area is calculates and also included some other parameter like skin threshold value and golden ratio. Depending upon values of these parameter the algorithm decide that it is face region or not. The algorithm is able to detect faces at different scales in a given video, as well as slightly tilted faces. Detected skin area is decided whether it is face or not.

\section{REFERENCES}

[1] MA Xinjun*, ZHANG Hongqiao, ZHANG Xin, Harbin Institute of Technology Shenzhen Graduate School, 518055,"A Face Detection Algorithm Based on Modified Skin-color Model" July 26-28, 2013, Xi'an, China, pp.3896-3900, Shenzhen, China.

[2] Zafar G. Sheikh, V. M. Thakare, S. S. Sherekar, 2012 2nd IEEE International Conference on Parallel, Distributed and Grid Computing "Advances in Face Detection Techniques in Video" SGB Amravati University, Amravati (M.S.), India.

[3] S. Stein and G. A. Fink, "A new method for combined face detection and identification using interest point descriptors," in Proc. IEEE Int. Conf. Autom. Face Gesture Recognit. Workshops, Mar. 2011, pp. 519-524.

[4] J. Qiang-rong and L. Hua-lan, 2010, "Robust Human Face Detection in Complicated Color Images", Proc. 2010 the 2nd IEEE International Conference on Information Management and Engineering (ICIME), pp.218 - 221, Chengdu, China.

[5] H. Guo, Y. Yu and Q. Jia, 2010, "Face Detection With Abstract Template", Proc.2010 3rd International Congress on Image and Signal Processing (CISP2010), pp.129-134, Yantai, China.

[6] Z. Li, L. Xue and F. Tan, 2010, "Face Detection In Complex Background Based On Skin Color Features And Improved Adaboost Algorithms", Proc. 2010 IEEE International Conference on Progress in Informatics and Computing (PIC), pp.723 -727, Shanghai, China.

[7] Boccignone, G., Chianese, A., et al. (2005). Foveated shot detection for video segmentation. IEEE transactions on circuits system and video technology, 15(3), 365-377.

[8] K. Seo, W. Kim, C. Oh and J. Lee, 2002, "Face Detection And Facial Feature Extraction Using Color Snake", Proc. ISIE 2002 - 2002 IEEE International Symposium on Industrial Electronics, pp.457-462, L 'Aquila, Italy. 